Beziehnng zum Cin eol, und läfst sich als Terpenmonohydrat auffassen; es ist jedoch zur Zeit noch nicht möglich, eine Strukturformel für denselben aufzustellen.

In geringer Menge wurde ferner Essigsäure nachgewiesen.

\title{
Mitteilungen aus dem pharmaceutisch-chemischen Institut der Universität Marburg.
}

Von Ernst Schmidt.

\section{Uber Papaveraceenalkaloide.}

\section{(Zweite Mitteilung). ${ }^{1}$ \\ Die Alkaloide der Wurzeln von Stylophoron diphyllum.}

Von Dr. Friedrich Selle.

(Eingegangen den 2. I. 1890).

Stylophoron diphyllum. Syn. 2 Meconopsis diphylla seu Chelidonium diphyllum ist eine im nördlichen Amerika in schattigen Wäldern wachsende Pflanze aus der Familie der Papaveraceen. Von den in Europa einheimischen Gliedern dieser Familie ist sie am meisten mit Chelidonium verwandt. Genus Chelidonio affine, differt imprimis habitu et stigmate. ${ }^{3}$

Von den Alkaloiden dieser Papaveracee war bisher wenig bekannt. E. Schmidt isolierte aus der Wurzel ein Alkaloid, welches in seiner Krystallform und seinem Verhalten eine gewisse Ahnlichkeit mit dem Chelidonin zeigte. $\$$ Lloyd gewann ebenfalls aus der Wurzel ein Alkaloid, welches Eykman analysierte. Letzterer gelangte aber zu keinem befriedigenden Resultate, da einerseits die Menge des ihm zur Verfügung gestellten Alkaloides eine zu geringe war, andererseits die analytischen Daten der freien Base mit denen des Pt-Salzes nicht ubereinstimmten. Trotzdem sprach Eykman die Ansicht aus, dafs das Alkaloid der Wurzel von Stylophoron diphyllun identisch mit Chelidonin von der Formel $\mathrm{C}_{19} \mathrm{H}_{19} \mathrm{NO}_{5}$ sei. Letztere Formel steht jedoch im Widerspruch mit den Resultaten, welche A. Henschke5 in seiner ausführlichen

1 Vergl. diese Zeitschrift 1888, 623.

2 Decandolle, Prodromus Systematis regni vegetabilis.

${ }^{3}$ Bentham et Hoocker, genera plantarum.

4 Diese Zeitschrift 1888, 623.

5 Ibidem 1888, 624 bis 644. 
Arbeit aber das Chelidonin erhielt, indem derselbe durch die Analysen des Platin- und Goldsalzes, des salzsauren, salpetersauren, schwefelsauren Salzes, sowie der reinen, bei $125^{\circ}$ getrockneten Base zu dem Schlufs gelangte, dafs diesem Alkaloide die Formel $\mathrm{C}_{20} \mathrm{H}_{19} \mathrm{NO}_{5}+\mathrm{H}_{2} \mathrm{O}$ zuzuerteilen sei.

Verfasser analysierte daher zunächst sowohl das lufttrockene als auch das bei $125^{0}$ getrocknete Chelidonin, welches Herr Professor Dr. E. Schmidt die Gute hatte, ihm als notorisch reines, selbst dargestelltes Material zur Verfugung zu stellen. Das mir übermittelte Alkaloid bildete gut ausgebildete, wasserhelle, glasglänzende monokline Krystalle, welche nach dem Trocknen bei $100^{\circ}$, bei $135^{\circ} \mathrm{C}$. schmolzen.

Die Analyse ergab folgende Daten:

I. 0,2411 lufttrockene Substanz gaben: $0,5694 \mathrm{CO}_{2}, 0,122 \mathrm{H}_{2} \mathrm{O}$.

II. 0,2544 lufttrockene Substanz gaben: $0,6017 \mathrm{CO}_{2}, 0,131 \mathrm{H}_{2} \mathrm{O}$.

III. 0,2717 Substanz verloren bei $100^{\circ} 0,0096 \mathrm{H}_{2} \mathrm{O}=3,53$ Proz. $\mathrm{H}_{2} \mathrm{O}$, bis auf $125^{\circ}$ erhitzt in ganzen $0,0125 \mathrm{H}_{2} \mathrm{O}=4,60$ Proz. $\mathrm{H}_{2} \mathrm{O}$.

IV. 0,3484 Substanz gaben nach der Methode von Will und Varrentra p p 0,0918 $\mathrm{Pt}=0,013 \mathrm{~N}=3,73$ Proz. N.

Gefunden :

\section{I.}

C 64,40

$$
\text { II. }
$$

H 5,62

$\mathrm{N} \quad-$

$\mathrm{H}_{2} \mathrm{O} \quad-$
64,50

5,72

$-$

$-$
III.

$-$

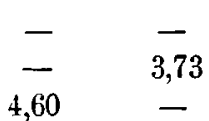

Berechnet für $\mathrm{C}_{20} \mathrm{H}_{19} \mathrm{NO}_{5}+\mathrm{H}_{2} \mathrm{O}$ : 64,70 Proz. 5,66 ” $3,77 \quad$ 4,85 ”

Da die vorstehenden Daten sehr gut mit den von Henschke ermittelten übereinstimmten, wurde von der bei 1250 getrockneten Substanz nur eine Analyse ausgeführt:

0,2592 der bei $125^{\circ}$ getrockneten Substanz gaben $0,6441 \mathrm{CO}_{2}$ $=67,77$ Proz. $\mathrm{C}$ und $0,128 \mathrm{H}_{2} \mathrm{O}=5,4$ Proz. $\mathrm{H}$.

$\begin{array}{ccc} & & \text { Berechnet für } \\ \text { Gefunden: } & \mathrm{C}_{20} \mathrm{H}_{10} \mathrm{NO}_{5}: \\ \text { C } & 67,77 & 67,98 \mathrm{Proz} \\ \mathrm{H} & 5,40 & 5,38,\end{array}$

Wie aus diesen Daten ersichtlich ist, stimmen meine Analysen mit denen von $A$. Henschke gut überein.

Auch die s. Z. von Will1 ansgeführten Analysen sind, wenigstens was den C- und H-Gehalt anbetrifft, mit der Formel $\mathrm{C}_{20} \mathrm{H}_{19} \mathrm{NO}_{5}+\mathrm{H}_{2} \mathrm{O}$ vereinbar.

Dieser Forscher führte drei Analysen des bei 1000 getrockneten

1 Annalen d. Chemie u. Pharm. 35, 114.

Arch. d. Pharm. XXVIIU. Bde. 3. Hent. 
Alkaloids aus (bei welcher Temperatur dasselbe zum gröfsten Teil sein Krystallwasser verliert), wobei er zn folgenden Resultaten gelangte:

\begin{tabular}{lrrc} 
& \multicolumn{1}{c}{ I. } & I. & III. \\
C & 69,07 & 68,76 & 68,30 Proz. \\
H & 5,62 & 5,65 & 5,69,
\end{tabular}

Berechnet man jedoch die von Will ermittelten Mengen der Kohlensäure und des Wassers nach dem gegenwärtig üblichen Atomgewicht, so ergeben sich die Zahlen:

$\begin{array}{crrcc} & & \text { Gefunden : } & & \text { Berechnet für } \\ & \text { I. } & \text { II. } & \text { III. } & \mathrm{C}_{20} \mathrm{H}_{19} \mathrm{NO}_{5}: \\ \text { C } & 68,14 & 67,83 & 67,37 & 67,98 \text { Proz. } \\ \mathrm{H} & 5,62 & 5,65 & 5,69 & 5,38 \text { n }\end{array}$

Auch der von Will bestimmte Pt-Gehalt des Pt-Doppelsalzes dürfte für die Formel $\mathrm{C}_{20} \mathrm{H}_{19} \mathrm{NO}_{5}$ sprechen, da zwischen den von ihm gefundenen Prozentzahlen des Pt-Gehalts: 1 17,42 und 17,60, und der für die obige Formel berechneten: 17,45, kein grofser Unterschied ist.

Nachdem so durch den Versuch von neuem festgestellt worden war, dafs dem Chelidonin die von A. Henschke aufgestellte Formel $\mathrm{C}_{20} \mathrm{H}_{19} \mathrm{NO}_{5}+\mathrm{H}_{2} \mathrm{O}$ zukommt, schien es weiter von Interesse zu sein, zu konstatieren, ob das in der Wurzel von Stylophoron diphyllum enthaltene Alkaloid die gleiche Zusammensetzung besitzt, bezüglich ob dasselbe, wie es nach den Vorversuchen von E. Schmidt den Anschein hat, mit dem Chelidonin identisch ist. Auf Veranlassung von Herrn Professor Dr E. Schmidt habe ich daher das fragliche Alkaloid, welches vor läufig mit dem Namen "Stylophorin" bezeichnet werden soll, aus Stylophoronwurzel in etwas gröfserer Menge dargestellt und dessen Zusammensetzung und Eigenschaften mit denen des Chelidonins verglichen.

\section{Darstellung.}

Zur Gewinnung der Stylophoronbasen wurden $5 \mathrm{~kg}$ grob gepulverter Stylophoronwurzeln mit schwefelsäurehaltigem Wasser wiederholt ausgekocht und die schleimige Masse durch ein grobmaschiges Sieb gegossen, da ein Kolieren durch Leinwand, Flanell etc. sich als unausfulhrbar erwies. Da die schleimigen Auszüge. auch bei längerem Stehen nicht absetzten, dagegen ein Vorversuch gelehrt hatte, dals durch Alhoholzusatz der Schleim abgeschieden werden konnte, dampfte ich die

1 Annalen d. Chemie u. Pharm. 35, 114. 
erhaltenen Auszüge auf den vierten Teil ihres Volumens ein und fugte dann soviel Alkohol zu, dafs nach dem Absetzen eine klare, leicht filtrierbare Flüssigkeit resultierte. Der durch Filtrieren getrennte Schleim wurde hierauf nochmals mit schwefelsäurehaltigem Alkohol ausgekocht und dieser Auszug nach dem Filtrieren mit dem ersteren vereinigt.

Dem mit Ammoniak alkalisch gemachten, nach dem Auskochen mit schwefelsäurehaltigem Alkohol verbliebenen Rtickstande konnte durch Alkohol, Äther, Chloroform, Petroleumäther etc. kein Alkaloid mehr entzogen werden. Zur Isolierung der Alkaloide wurden daher die sauren alkoholischen Extrakte durch Destillation von Alkohol befreit, die aus dem Destillationsrückstande ausgeschiedenen harzartigen Massen abfiltriert und das Filtrat mit Ammoniak übersättigt. Der bierdurch erzeugte Niederschlag wurde alsdann nach dem Auswaschen und Trocknen mit schwefelsäurehaltigem Alkohol ausgekocht, die filtrierten Auszuige nach Zusatz von Wasser von Alkohol befreit und der filtrierte Rückstand von neuem mit Ammoniak gefällt.

Das auf diese Weise gewonnene Präcipitat zeigte noch keineswegs einen einheitlichen Charakter, es wurde dasselbe daher, behufs weiterer Reinigung (zur Entfernung von Chelerythrin etc.), im lufttrockenen $\mathrm{Zu}$ stande mit Äther erschöpft, die hierdurch erzielten blau fluorescierenden Auszüge (A) verdunstet, die bei der Extraktion restierenden Rohalkaloide dagegen in wenig schwefelsäurehaltigem Wasser gelöst und die klare Lösung mit dem mehrfachen Volumen rauchender Salzsäure gemischt.

Es resultierte hierdurch eine reichliche krystallinische, jedoch immer noch braun gefärbte Abscheidung, welche nach mehrtägigem Stehen von der Mutterlange getrennt und auf porösen Thonplatten geprefst wurde. Die salzsaure Mutterlauge lieferte, nach abermaliger Ausfallung mit Ammoniak, unter den gleichen Versuchsbedingungen eine weitere, jedoch etwas heller gefärbte, krystallinische Ausscheidung. Aus den hierdurch gewonnenen Hydrochloriden wurde alsdann die freie Base durch Fällen der wässerigen Lösung mit Ammoniak abgeschieden, letztere, nach dem Auswaschen und Trocknen, in chloroformhaltigem Alkohol gelöst, diese Lösung mit frisch ausgeglühter Tierkohle digeriert und schliefslich der freiwilligen Verdunstung uberlassen.

Die allmählich ausgeschiedenen Krystalle, welche in ihrem Äufseren eine grofse Ähnlichkeit mit Chelidonin zeigten, liefsen zwar in ihrer krystallographischen Ausbildung nichts zu wünschen ubrig, jedoch besafsen sie noch gelbe Farbe. Letztere liefs sich auch durch wieder- 
holtes Umkrystallisieren aus den gleichen Lösungsmitteln nicht vollständig entfernen, ebensowenig durch Behandlung der essigsauren wässerigen Lösung mit Bleiacetat und Schwefelwasserstoff. Dagegen gelang es das gesuchte Alkaloid in chemischer Reinheit dadurch zu erhalten, dafs die alkoholische Lösung desselben mit Salzsäuregas gesättigt, aus dem abgeschiedenen Hydrochlorid dann die freie Base isoliert und letztere hierauf von neuem in der gleichen Weise in das Hydrochlorid übergeführt wurde. Die aus dem wiederholt gereinigten Hydrochlorid durch Fällung mit Ammoniak erzielte Base bildete schliefslich ein weifses, krystallinisches Pulver, welches durch Lösen in chloroformhaltigem Alkohol und freiwilliges Verdunstenlassen dieser Lösung leicht in ansehnliche, vollkommen einheitliche Krystalle verwandelt werden konnte.

Eigenschaften. Das in der angegebenen Weise gewonnene Stylophorin bildete ansehnliche, wasserhelle, glasglänzende Krystalle; welche in dem $\ddot{A}$ ufseren und in den Löslichkeitsverhältnissen in jeder Beziehung mit dem Chelidonin iibereinstimmten. Den Schmelzpunkt des Stylophorins fand ich nach dem Trocknen bei $100^{\circ}$ bei $135^{\circ} \mathrm{C}$. E. Schmidt und Eykman ermittelten den Schmelzpunkt bei der gleichen Temperatur. Das Gleiche gilt fiir das Chelidonin.

Gegen Alkaloidreagentien zeigte das Stylophorin in essigsaurel Lösung das folgende, dem Chelidonin durchaus entsprechende Verhalten:

$$
\text { Lösung } 1: 100 .
$$

$\begin{array}{lll}\text { Wismutjodidjodkalium } & =\text { weifse Fällung, } \\ \text { Quecksilberjodidjodkalium } & =" " \\ \text { Phosphomolybdänsäure } & = & " \\ \text { Gerbsäure } & = & " \\ \text { Jodjodkalium } & =\text { gelbbraune " } \\ \text { Kaliumcadmiumjodid } & =\text { weifse " } \\ \text { Phosphowolframsäure } & =" " \\ \text { Pikrinsäure } & =\text { gelbe } \\ \text { Platinchlorid } & =\text { gelbweifse " } \\ \text { Goldchlorid } & =\text { orangerote " } \\ \text { Quecksilberchlorid } & =\text { erst Trüb., dann flock. Niederschl., } \\ \text { Bleiessig } & =\text { weifse Fällung, } \\ \text { Chromsaures Kalium } & =\text { gelber Niederschlag, } \\ \text { Bromwasser } & =\text { gelbe Fällung. }\end{array}$


F. Selle, Die Alkaloide der Wurzeln von Stylophoron diphyllum. 101

Das Alkaloid in Substanz zeigte nachstehende Reaktionen:

Konzentrierte Schwefels. $=$ gelb, braun, kirschr.u.schliefs!.viol.

Froebde's Reagens

Erdmann's Reagens

$\mathrm{H}_{2} \mathrm{SO}_{4}+\mathrm{K}_{2} \mathrm{Cr}_{2} \mathrm{O}_{7}$

Vanadinschwefelsïure
= dunkelgrün, dann blaugrün,

$=$ grün längere Zeit, dann vom

Rande her gelb werdend,

$=$ grasgrün, dann blaugrün, schliefs-

lich schmutzig grïn,

$=$ hellgrün, dunkelgrün, blaugrün, schliefslich schmutzig grün,

Konzentr. Salpetersäure $=$ gelb.

Die Farbenreaktionen wurden hier, wie auch bei den später beschriebenen Alkaloiden, in der Weise ausgeführt, dafs eine Spur des zu einem feinen Pulver verriebenen Alkaloids mit 2 Tropfen des betreffenden Reagens auf einem Uhrglase zusammengebracht wurde. Unter bestiindigem Umrühren mit einem Glasstabe wurden dann die Farbenerscheinungen resp. -veränderungen notiert. Wendet man ganze Krystalle an oder unterläfst man das Umrühren, so kommt man zu wesentlich anderen Resultaten. Obige Reaktionen wurden mit dem Chelidonin sowie dem Stylophorin zu gleicher Zeit angestellt, und bei beiden Basen waren die Ergebnisse vollständig dieselben. Zu bemerken ist noch, dafs ich, entgegen der Angabe von A. Henschke,1 Brom wirke auf Chelidonin nicht ein, gefunden habe, dafs, wenn man $z u$ der in $\mathrm{HCl}$ oder $\mathrm{C}_{2} \mathrm{H}_{4} \mathrm{O}_{2}$ bewirkten Lösung nach dem Erkalten Bromwasser gilut, ein schön gelber Niederschlag erhalten wird.

Es liefert also auch das Chelidonin wie viele andere Alkaloide mit Brom ein Substitutions- bezüglich ein Additionsprodnkt,

Dis Analysen des Stylophorins lieferten folgende Daten:

$0,1735 \mathrm{~g}$ der lufttrockenen Base verloren bei $12500,0082 \mathrm{~g}$ an Gewicht.

$$
\begin{aligned}
& \text { Gefunden: Berechnet für } \mathrm{C}_{20} \mathrm{H}_{19} \mathrm{NO}_{5}+\mathrm{H}_{2} \mathrm{O} \text { : } \\
& \mathrm{H}_{2} \mathrm{O} \text { 4,72 Proz. 4,85 Proz. }
\end{aligned}
$$

I. 0,1842 der lufttrockenen Substanz gaben 0,4348 Kohlensäure und 0,859 Wasser.

II. 0,4654 der lufttrockenen Substanz lieferten nach der Methode von Will und Varrentrapp 0,119 Platin $=0,01687$ Stickstoff.

1 Diese Angabe bezieht sich nur auf das Verhalten des Chelidonins in Chloroformlösung gegen Brom (s. Inaugural-Dissertation). E. S. 


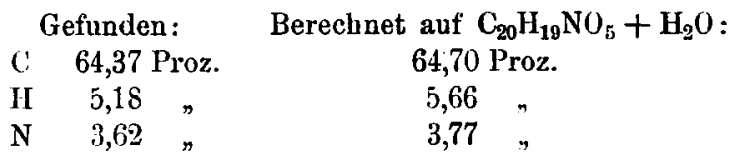

III. 0,1672 der bei $125^{\circ}$ getrockneten Substanz gaben 0,4156 Kohlensäure und 0,0898 Wasser $=67,79$ Proz. Kohlenstoff und 5,56 Proz. Wasserstoff.

$$
\begin{gathered}
\text { Berechuet auf } \mathrm{C}_{30} \mathrm{I}_{19} \mathrm{NO}_{5}: \\
\mathrm{C}=67,98 \text { Proz. } \quad \mathrm{HI}=5, ; 88 \text { Proz. }
\end{gathered}
$$

Die gefundenen Werte dürften mit den für die Formel $\mathrm{C}_{20} \mathrm{H}_{19} \mathrm{NO}_{5}+\mathrm{H}_{2} \mathrm{O}$ lerechneten Prozentzahlen wohl vereinbar sein.

\section{Stylophorin-Goldchlorid.$$
\mathrm{C}_{20} \mathrm{H}_{19} \mathrm{NO}_{5} \mathrm{HCl}, \mathrm{AuCl}_{3} \text {. }
$$

Salzsaures Stylophorin wurde zur Darstellung dieses Doppelsalzes in heifsem Wasser gelöst, die Lösung mit wenigen Tropfen Salzsäurc angesäuert und mit Goldchloridlösung im Überschuls versetzt. Es tiel hierdurch ein orangerot gefärbter, flockiger Niederschlag, welcher sich nach dem Auswaschen und Trocknen als löslich in Alkohol erwies. Die gesamte Menge des Niederschlages wurde daher in Alkohol gelöst. Nach dem Erkalten schieden sich dunkelrote, zu Büscheln vereinigte nadelförmige Krystalle aus. Bei $100^{0}$ getrocknet verloren dieselben kein Krystallwasser.

I. 0,2236 des bei $100^{\circ}$ getrockneten Salzes gaben beim Glühen bis zum konstanten Gewicht $0,0635 \mathrm{Au}$.

II. 0,1706 Substanz gaben $0,1423 \mathrm{AgCl}=0,035$ Cl.

III. 0,2236 Substanz gaben nach dem Verbrennen $0,2844 \mathrm{CO}_{2}$ und $0,0629 \mathrm{H}_{2} \mathrm{O}$.

JV. 0,1831 Substanz gaben nach dem Verbrennen $0,2300 \mathrm{CO}_{2}$ und $0,0521 \mathrm{H}_{2} \mathrm{O}$.

\begin{tabular}{cccccc} 
& \multicolumn{3}{c}{ Gefunden: } & \multicolumn{3}{c}{ Bercehnet für } \\
& I. & II. & III. & IV. & $\mathrm{C}_{30} \mathrm{H}_{19} \mathrm{NO}_{5} \mathrm{HCl}, \mathrm{AuCl}_{3}$ : \\
$\mathrm{C}$ & 34,68 & 34,28 & - & - & 34,64 Proz. \\
$\mathrm{H}$ & 3,12 & 3,16 & - & - & $2,88 \% "$ \\
$\mathrm{Cl}$ & - & - & 20,51 & - & $20,49 "$ \\
$\mathrm{Au}$ & - & - & - & 28,39 & $28,39 "$
\end{tabular}

\section{Stylophorin-Platinchlorid.$$
\left[\mathrm{C}_{20} \mathrm{H}_{19} \mathrm{NO}_{5} \mathrm{HCl}\right]_{2} \mathrm{PtCl}_{4}+2 \mathrm{H}_{2} \mathrm{O} \text {. }
$$

Dieses Salz wurde analog dem Goldsalz durch Fällen der beifsen Lösung des $\mathrm{HCl}$-Salzes mit überschlissigem Platinchlorid dargestellt. Der entstandene Niederschlag war von gelblich-weifser Farbe. Aus Alkohol war dieses Salz nicht krystallisiert zu erhalten. 
F. Selle, Die Alkaloide der Wurzeln von Stylophoron diphyllum.

I. 0,2361 des lufttrockenen Salzes verloren bei $100^{\circ}$ $0,0087 \mathrm{H}_{2} \mathrm{O}=3,68$ Proz. $\mathrm{H}_{2} \mathrm{O}$.

Berechnet für $\left[\mathrm{C}_{20} \mathrm{H}_{19} \mathrm{NO}_{5} \mathrm{BCl}\right]_{2} \mathrm{PtCl}_{4}+2 \mathrm{H}_{2} \mathrm{O}$ : $\mathrm{H}_{2} \mathrm{O} \quad 3,10$ Proz.

II. 0,2274 des bei $100^{\circ}$ getrockneten Salzes gaben nach dem Glühen bis zum konstanten Gewicht 0,0394 Pt.

III. 0,1557 des bei $100^{\circ}$ getrockneten Salzes gaben bei der Verbrennung mit Bleichromat und vorgelegter reduzierter Kupferspirale $0,2442 \mathrm{CO}_{2}$ und $0,0551 \mathrm{H}_{2} \mathrm{O}$.

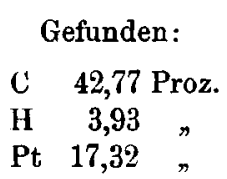

Berechnet für

$\left[\mathrm{C}_{20} \mathrm{H}_{19} \mathrm{NO}_{5} \mathrm{HCl}_{2} \mathrm{PtCl}_{4}\right.$ : 43,03 Proz. 3,60 17,45 ,

\section{Salzsaures Stylophorin.}

Dieses Salz wurde gewonnen durch Einleiten von $\mathrm{HCl}$-Gas in die alkoholische Auflösung der freien Base und Umkrystallisieren des Niederschlages aus heifsem Wasser. Es bildet bei langsamer Abkühlung des Lösungsmittels feste, würflige Krystalle, welche in Wasser schwer lüslich sind. Bei $100^{\circ}$ getrocknet verliert dasselbe kein Wasser.

I. 0,1863 Substanz gaben $0,0671 \mathrm{AgCl}=0,0170 \mathrm{HCl}$.

II. 0,3627 Substanz gaben bei der Verbrennung mit Bleichromat und vorgelegter reduzierter Kupferspirale $0,8139 \quad \mathrm{CO}_{2}$ und $0,1779 \mathrm{H}_{2} \mathrm{O}$.

Gefunden: Berechnet für $\mathrm{C}_{20} \mathrm{H}_{19} \mathrm{NO}_{5} \mathrm{HCl}$ :

$\begin{array}{lcll}\text { C } & 61,20 & \text { Proz. } & 61,61 \text { Proz. } \\ \text { H } & 5,44 & 5,13 & \\ \text { HCl } & 9,12 & & 9,37\end{array}$

\section{Salpetersaures Stylophorin.}

Das Nitrat wurde dargestellt durch Auflösen des Alkaloids in heifsem Alkohol und Versetzen der alkoholischen Lösung mit verdunnter Salpetersäure bis zur sauren Reaktion. Die gewonnenen farblosen sänlenförmigen Krystalle verloren bei 1000 getrocknet nichts an Gewicht. Eine Elementaranalyse ergab für $\mathrm{C}$ und $\mathrm{H}$ folgende Resultate:

$0,1762 \mathrm{~g}$ gaben $0,3727 \mathrm{CO}_{2}$ und $0,081 \mathrm{H}_{2} \mathrm{O}=57,68 \mathrm{Proz}$. $\mathrm{C}$ und 5,10 Proz. $\mathrm{H}$.

Berechnet für $\mathrm{C}_{20} \mathrm{H}_{19} \mathrm{NO}_{5} \mathrm{HNO}_{3}$ :

$$
\begin{array}{cc}
\text { C } & 57,69 \text { Proz. } \\
\text { H } & 4,80,
\end{array}
$$


Es mag hier zur besseren Übersicht eine Zusammenstellung der von Will, Eykman, A. Henschke (l. c.) für Chelidonin ermittelten Werte, sowie der vom Verfasser für das Chelidonin und Stylophorin erzielten Resultate folgen :

\begin{tabular}{|c|c|c|c|c|c|c|}
\hline & Vill & Eykman & A.Henschke & \multicolumn{2}{|c|}{ Verfasser } & Berechnet \\
\hline & & \multicolumn{4}{|c|}{ Lufttrockene Substanz. } & \\
\hline & & \multicolumn{2}{|c|}{ Chelidonin } & Chelidon. & Stylophor. & $\mathrm{C}_{20} \mathrm{H}_{10} \mathrm{NO}_{5}+\mathrm{H}_{2} \mathrm{O}$ \\
\hline & & \multicolumn{2}{|c|}{ Im Mittel: } & Im Mittel: & & \\
\hline C & - & 63,36 & - & 64,45 & 64,37 & 64,70 \\
\hline $\mathrm{H}$ & - & 5,68 & - & 5,18 & 5,18 & 5,66 \\
\hline $\mathrm{N}$ & - & 4,09 & -1 & 3,75 & 3,62 & 3,77 \\
\hline & & \multicolumn{4}{|c|}{ Getrocknete Substanz. } & \multirow{3}{*}{$\mathrm{C}_{30} \mathrm{H}_{19} \mathrm{NO}_{5}$} \\
\hline 10 & $00^{\circ}$ & & $125^{\circ}$ & $125^{\circ}$ & $125^{\circ}$ & \\
\hline $\operatorname{In} \mathrm{M}$ & Mittel: & & Im Mittel: & & & \\
\hline C 6 & $67,78^{\circ}$ & - & 67,79 & 67,77 & 67,79 & 67,98 \\
\hline & 5,65 & - & 5,48 & 5,40 & 5,56 & 5,38 \\
\hline $\mathbf{N} 1$ & $12 ?$ & - & 3,85 & - & - & - \\
\hline \multirow{2}{*}{\multicolumn{2}{|c|}{$\mathrm{H}_{2} \mathrm{O} 4,8$}} & - & 4,80 & 4,60 & - & - \\
\hline & & \multicolumn{5}{|c|}{ Platin-Doppelsalz, bei $100^{\circ},\left(\mathrm{C}_{20} \mathrm{H}_{19} \mathrm{NO}_{5} \mathrm{HCl}\right)_{2} \mathrm{PtCl}_{4}$. } \\
\hline $\mathbf{P t}$ & 17,5 & 17,48 & $\left|\begin{array}{c}\text { Im Mittel : } \\
17,48\end{array}\right|$ & - & 17,32 & 17,45 \\
\hline$C^{\prime}$ & - & - & 42,87 & - & 42,77 & 43,03 \\
\hline $\mathrm{H}$ & - & - & 4,04 & - & 3,93 & 3,60 \\
\hline$N^{-}$ & - & - & 2,48 & 一 & - & 2,42 \\
\hline \multirow[t]{2}{*}{$\mathrm{H}_{2} \mathrm{O}$} & - & 3,60 & 4,11 & - & 3,83 & 3,10 \\
\hline & & \multicolumn{5}{|c|}{$\begin{array}{l}\text { Gold-Doppelsalz, } \mathrm{C}_{20} \mathrm{H}_{10} \mathrm{NO}_{5} \mathrm{HClAuCl} \text {, krystallisiert. } \\
\text { Verliert bei } 100^{\circ} \text { kein Wasser. } \\
\text { Im Mittel: }\end{array}$} \\
\hline $\mathrm{C}$ & - & - & 34,40 & - & 34,48 & 34,64 \\
\hline $\mathrm{H}$ & - & - & 3,01 & - & 3,14 & 2,88 \\
\hline Cl & - & - & 20,35 & 一 & 20,51 & 20,49 \\
\hline \multirow[t]{2}{*}{$\mathrm{Au}$} & - & - & 28,21 & - & 28,39 & 28,39 \\
\hline & & \multicolumn{5}{|c|}{$\begin{array}{l}\text { Salzsaures Salz, } \mathrm{C}_{20} \mathrm{H}_{10} \mathrm{NO}_{5} \mathrm{HCl} \text {. } \\
\text { Im Mittel. }\end{array}$} \\
\hline C & - & - & 61,46 & - & 61,20 & 61,61 \\
\hline $\mathrm{H}$ & - & - & 5,25 & - & 5,44 & 5,13 \\
\hline \multirow[t]{2}{*}{$\mathrm{HCl}$} & - & - & 9,12 & - & 9,12 & 9,37 \\
\hline & & \multicolumn{5}{|c|}{$\begin{array}{l}\text { Salpetersaures Salz, } \mathrm{C}_{20} \mathrm{H}_{19} \mathrm{NO}_{5} \mathrm{HNO}_{3} \text {. } \\
\text { Im Mittel: }\end{array}$} \\
\hline C & 一 & - & 57,69 & - & 57,68 & 57,69 \\
\hline$H$ & - & - & 4,80 & - & 5,10 & 4,80 \\
\hline
\end{tabular}


Aus vorstehenden Daten dürfte zur Genüge hervorgehen, dafs das Stylophorin mit dem Chelidonin identisch ist. Die Identität ergibt sich nicht allein durch das chemische Verhalten, sondern auch durch die Übereinstimmung in der Krystallform. Herr Professor Dr. Lu e decke, welcher die Güte hatte, die Krystalle des Stylophorins zu messen, teilt mir darüber folgendes mit:

Das Stylophorin stimmt mit dem früher gemessenen Chelidonin (s. diese Zeitschrift 1888, p. 626) in den Winkeln, in der Säulenform und der Zone $p: c$, also Săule zur Pyramide zur Basis vollkommen überein.

$\begin{array}{ccc}\begin{array}{c}\text { Chelidonin } \\ \text { gemessen : }\end{array} & \begin{array}{c}\text { Chelidonin } \\ \text { berechnet : }\end{array} & \begin{array}{c}\text { Stylophorin } \\ \text { gemessen: }\end{array} \\ \overline{1} 10: \overline{1} 11=31034^{\prime} & 31031,5^{\prime} & 31031,5^{\prime} \\ \overline{1} 11: 001=60^{\circ} 58^{\prime} & - & 60^{\circ} 21^{\prime} \\ \overline{1} 11: 001=60^{\circ} 58^{\prime} & - & 60^{\circ} 38^{\prime} \\ 001: 1 \overline{1} 0=87012^{\prime} & 87030,5^{\prime} & 87049^{\prime}\end{array}$

In 001 liegen die Auslöschungen, der monosymmetrischen Symmetrie entsprechend, diagonal.

A. Henschke (l. c.) fand, dafs das Chelidonin bei der Oxydation mit Kaliumpermanganat in alkalischer Lösung ein ähnliches Verhalten wie Morphin zeigt, indem dabei nur Kohlensäure, Oxalsäure, Methylamin und Ammoniak gebildet werden. Hierdurch veranlafst, versuchte ich, ob das Chelidonin anch durch Behandeln mit Salzsäure vielleicht ein ähnliches Zersetzungsprodukt liefert, wie das Morphin. Zu diesem Zweck wurden einige Gramm krystallisierten Chelidonins (Stylophorins) mit rauchender Salzsäure im zugeschmolzenen Rohre drei Stunden lang auf $150^{\circ}$ erhitzt. Es scheint jedoch hierdurch eine nahezu vollständige Zerstörung des Alkaloids veranlafst zu werden, denn der Inhalt der Röhre bestand aus einer schwarzen, kohligen Masse, der durch verschiedene Lösungsmittel nichts entzogen werden konnte, ebenso lieferte die Salzsäure nach dem Verdünnen mit Wasser und Versetzen mit Ammoniak nur noch einen geringen Niederschlag. Beim Erhitzen des Chelidonins (Stylophorins) mit Salzshure im zugeschmolzenen Rohre auf $100^{\circ}$ wurde letzteres nicht ganz gelöst. Die Salzsäure hatte eine grüne Farbe angenommen, die aber nach dem Verdünnen der Flüssigkeit mit Wasser unter Bildung eines geringen, braunen Bodensatzes nach einiger Zeit verschwand. Das Volumen der nicht in Lösung gegangenen 
Krystalle hatte sich vergröfsert; dieselben wurden zur näheren Charakterisierung in viel Wasser, dem etwas Salzsäure zugesetzt war, durch Kochen gelöst und die Lösung mit Ammoniak behandelt. Nach Auflösung des getrockneten Niederschlages in chloroformhaltigem Alkohol und Verdunsten der Flïssigkeit schieden sich nur Chelidoninkrystalle aus, erkennbar durch Form und Schmelzpunkt. Unter letzteren Bedingungen scheint somit das Chelidonin durch Salzsäure nicht zersetzt zu werden. Wenn auch dieser Versuch nicht den Erfolg hatte, eine Analogie zwischen Chelidonin und Morphin darzuthun, so scheinen doch die physiologischen Eigenschaften, welche Herr Professor Dr. Hans Meyer festzustellen die Liebenswürdigkeit hatte, auf eine gewisse Verwandtschaft des Chelidonins mit dem Morphin hinzudeuten. Das Ergebnis der physiologischen Untersuchung war, nach gütiger Mitteilung von Herrn Professor Dr. H. Me yer, folgendes:

„Chelidoninum sulfuricum, in 5proz. Lösung verwandt, bewirkt in Dosen von einigen Centigrammen an Fröschen centrale Lähmung, die sich anfangs in schlaffen, schlecht koordinierten Bewegungen, schliefslich in völliger Unfähigkeit zu spontaner Bewegung äufsert. Die Erregbarkeit der motorischen Muskeln bleibt dagegen intakt. Die Reaktion gegen chemische, später auch gegen taktile, Reize nimmt erheblich ab, um schliefslich ganz zu schwinden.

In diesem Zustande liegen die Tiere vollkommen geliihmt und reaktionslos da, nur die Thätigkeit des Herzens und der Respiration dauert einstweilen fort. Bei Säugetieren kommt es nach Applikation von 4 bis $6 \mathrm{cg}$ ebenfalls zu grofser Unsicherheit der Bewegungen, sowie zu einer Art Narkose: die Empfindlichkeit ist stark vermindert; Nadelstiche, Kniffe, Schnitte werden nicht beantwortet, auch lälst sich das vergiftete Tier ohne Widerstand in unnatürliche Lage bringen und verharrt in derselben längere Zeit; labei scheint die Reflexfunktion des Rückenmarks erhalten zu sein.

Stärkere taktile Reize rufen in gewohnter Weise Reflexbewegungen hervor. Der ganze Zustand gleicht in vieler Beziehung der Morphinnarkose; auch die Herzthätigkeit scheint in ähnlicher Weise beeinflufst «u werden. Die Zahl der Pulse nimmt erheblich ab, besonders stark bei Fröschen und Kaninchen; bei Katzen in viel geringerem Grade. An Menschen konnten entscheidende Versuche noch nicht angestellt werden. Aufser den centralen Lähmungserscheinungen bewirkt das Chelidonin aber auch eine Laihmung der peripheren sensiblen Nervenapparate. Taucht 
man ein Bein eines Frosches in 5 proz. Chelidoninlösung, so wird dasselbe vollkommen insensibel, während die motorische Erregbarkeit normal bleibt.

Werden einige Tropfen der Lösung in den Bindehautsack am Auge eines Kaninchens oder Meerschweinchens injiziert, so wird nach einigen Minuten die Cornea für Stiche, oberflächliche Schnitte und dergleichen unempfindlich. Auch bein Menschen tritt ohne vorangehende Reizung eine, allerdings nur geringe, Abstumpfung der Corneal-Empfindlichkeit ein. Vollständige Anästhesierung zum $Z$ wecke schmerzfreier Operationen läfst sich jedoch mit Chelidonin nicht erzielen."

Da die Einwirkung der Salzsäure auf Chelidonin resultatlos verlief, so wurde das Verbalten dieser Base gegen Jodwasserstoff untersucht.

Nach der von Zeisel angegebenen Methode im Glycerinbade mit Jodwasserstoffsäure erhitzt, wurde kein Jodmethyl gebildet. Die vorgelegte alkoholische Silbernitratlösung wurde kaum merklich getrübt. Die Base enthält somit keine Methoxylgruppe. Es wlirde dies Verhalten der Vermutung von A. Henschke (l. c.) widersprechen, nach welcher das Chelidonin vielleicht als ein Methoxylchelerythrin aufzufassen ist, eine Vermutung, zu der allerdings unter allem Vorbehalt nur der Umstand Veranlassung gab, dafs die F'ormel des Chelidonins $\mathrm{C}_{20} \mathrm{H}_{19} \mathrm{NO}_{5}$ sich durch einen Mehrgehalt von $\mathrm{CH}_{2}$. $\mathrm{OH}$ von der von $\mathrm{S}$ chiel für das Chelerythrin aufgestellten Formel $\mathrm{C}_{19} \mathrm{H}_{17} \mathrm{NO}_{4}$ unterscheidet.

Das Stylophorin (Chelidonin) ist zwar das hauptsächlichste, keineswegs jedoch aber das einzige Alkaloid, welches in der Wurzel von Stylophoron diphyllum vorkommt; in geringer Menge finden sich vielmehr darin wenigstens noch zwei andere, von dem Chelidonin wesentlich verschiedene Basen. Bei der Schwierigkeit, das zu einer eingehenderen Untersuchung letzterer Alkaloide erforderliche Rohmaterial zu beschaffen, mufs ich mich leider vorlüufig auf wenige Bemerkungen hiertiber beschränken. Sobald jedoch gröfsere Mengen Stylophoronwurzeln zu einem civilen Preise erhältlich sein werden, soll die Ontersuchung auch dieser Basen, die dem Anschein nach in Beziehung zu den in den Wurzeln von Chelidonium majus und Sanguinaria canadensis enthaltenen Alkaloiden stehen, von neuem in Angriff genommen werden.

Zur Isolierung anderweitiger Alkaloide diente der beim Verdunsten des ätherischen Auszuges der Rohalkaloide (A, s. S. 99) verbleibende harzartige Rückstand. Letzterer löste sich bei der Digestion mit verdünnter Salzsäure nur teilweise, unter Zuricklassung eines brännlichen Harzes (B), auf. Aus der erzielten, intensiv rot getärbten Lösung 
schieden sich beim Kochen weifsliche Krystalle aus, welche im Vereine mit jenem ungelöst gebliebenen Harze (B) durch Kochen mit viel Wasser, dem etwas Salzsăure zugefügt war, nach Möglichkeit gelöst wurden. Ammoniak rief in dieser Lösung eine flockige Abscheídung hervor, welche nach dem Auswaschen und Trocknen durch Lösen in chloroformhaltigem Alkohol und freiwilliges Verdunstenlassen dieser Lösung zur Krystallisation gebracht werden konnten. Die auf diese Weise gewonnenen Krystalle zeigten jedoch keinen einheitlichen Charakter, da neben Stylophorin (Chelidonin), welches sich durch die Form und durch den Schmelzpunkt kennzeichnete, auch nadelförmige Krystalle abgeschieden waren. Letztere wurden ausgelesen und aus Essigäther umkrystallisiert. Es resultierten hierdurch nadelförmige, fast farblose, bei 193 bis $1950 \mathrm{C}$. schmelzende Krystalle, welche sich in verdünnter Salzsiiure als nahezu unlöslich, in verdünnter Schwefelsäure als schwer löslich erwiesen. Aus letzterer Lüsung schied sich das gebildete Sulfat in Nadeln aus. Die Menge dieses Alkaloids reichte zu einer näheren analytischen Untersuchung nicht aus, ich mufste mich daher zunächst darauf beschrinken, das Verhalten dieser Base gegen Alkaloidreagentien zu studieren. In essigsaurer Lösung zeigte dieselbe folgendes Verhalten :

$\begin{array}{ll}\text { Wismutjodidjodkalium } & =\text { rotgelbe Fällung, } \\ \text { Gerbsäure } & =\text { keine } \\ \text { Phosphowolframsäure } & =\text { weifse } \\ \text { Kaliumcadmiumjodid } & =", \\ \text { Phosphomolybdänsäure } & =\text { weifsgelbe " " } \\ \text { Quecksilberjodidjodkalium } & =\text { weifse, käsige Fällung, } \\ \text { Kaliumchromat } & =\text { gelbe Fällung, } \\ \text { Bromwasser } & =\text { gelbe Trübung, dann gelbe, käsige } \\ & \text { Flocken. }\end{array}$

Ein Gemisch der Auflösungen von Eisenchlorid und Ferricyankalium wurde nicht verändert, erst nach einiger Zeit wurde die Flüssigkeit grtin gefärbt. Mit konzentrierter Schwefelsăure wurde das Alkaloid vorübergehend gelb, blalsgrün, dann schwach violett, zuletzt braun.

Salpetersäure rief nur eine Gelbfirbung hervor, die sich nicht veränderte.

Froede'sches Reagens: gelb, griun, blaugrlin, intensiv blau, schliefslich lange Zeit blaugriun. 
Erdmann'sches Reagens: Gelb, prächtig grün, vom Rande her vorübergehend blau, dann schmutzig grün.

Vanadin-Schwefelsäure: grün, blaugrün, lange Zeit sehr schön blau, dunkel blangrün.

Der durch Kaliumchromat in der Alkaloidlösung erzeugte Niederschlag zeigte nach dem Auswaschen gegen konzentrierte Schwefelsäure folgendes Verhalten: Es wurde sofort eine schön grüne Färbung hervorgerufen, welche später in braun überging.

Die intensiv rot gefärbte Flüssigkeit, welche die in Salzsïure leicht löslichen Körper des ätherischen Auszuges der Rohalkaloide (A) enthalten konnte, wurde mit Ammoniak versetzt und der abgeschiedene braune Niederschlag nach dem Auswaschen und Trocknen hierauf mit Äther im Soxhlet'schen Apparate extrahiert. Der Niederschlag löste sich hierbei nur zum Teil zu einem blau fluorescierendem Liquidum, aus welchem trockener Chlorwasserstoff eine intensiv rot gefirbte, krystallinische Masse ausschied, die aus Wasser in schön rot gefärbten, den Hydrochloriden des Chelerythrins und Sanguinarins sehr ähnlichen Nadeln krystallisierte. Ob cliese Krystalle zu den Salzen letzterer Alkaloide in Beziehung stehen oder vielleicht mit dem einen oder anderen derselben identisch sind, habe ich vorläufig aus Mangel an Material nicht entscheiden können. Es war dies um so weniger möglich, als das Sanguinarin von Naschold (Journ. f. prakt. Chemie 106, 385) nach den Untersuchungen von Herrn G. König (Privatmitteilung) aus einem Gemisch mehrerer Alkaloide besteht.

\section{Mitteilung aus dem pharmaceutischen Institut der Universität zu Breslau. Drei nene Bleiverbindungen.}

Von Dr. Georg Karsner.

(Eingegangen den 9. XII. 1889.)

In dem Bestreben, einen Ersatz für das von mir frliher als Oxydationsmittel vorgeschlagene mangansaure Baryum aufzufinden, habe ich versucht, neue Verbindungen herzustellen, oder aber eine neue Kombination bekannter Verbindungen zu gewinnen, durch welche es mbglich 